Session 1520

\title{
Intelligent Diabetes Management
}

\author{
Mario A Garcia, Carl Steidley, Maruthi Dantu, Rui Shen \\ Texas A\&M University Corpus Christi
}

\begin{abstract}
Diabetes is a disease that affects many people in the world ${ }^{1}$. Diabetes cost in the U.S.A. is estimated to represent $5.8 \%$ of total personal health-care expenditures. In Europe diabetes is estimated to consume about $10 \%$ of the total health care budget. An Intelligent Diabetes Management System was developed by computer science students at Texas A\&M University Corpus Christi to help people monitor and control the blood glucose level. The diabetes experts were Dr. Steve Ponder and Dr. Hilda Ramirez from Driscoll Children's Hospital. This paper describes the steps followed to implement it.
\end{abstract}

\section{Introduction}

Diabetes is a disease that affects more than 100 million people ${ }^{1}$. Diabetes cost in the U.S.A. is estimated to represent $5.8 \%$ of total personal health-care expenditures. In Europe diabetes is estimated to consume about $10 \%$ of the total health care budget. Diabetes Mellitus (technical term for Diabetes) is a chronic condition associated with abnormally high levels of glucose(sugar) in the blood. The food that a person intakes is converted into glucose, which is used as a source of energy. A hormone called Insulin, secreted by the pancreas helps to disintegrate glucose so the cells can absorb it. When a person has diabetes, the body either doesn't generate enough insulin or can't use its own insulin as it should. This causes sugars to build up in blood. Diabetes can cause serious health complications like heart disease, blindness, kidney failure, and lower-extremity amputations.

Diabetes is the seventh leading cause of death in the United States. Symptoms of diabetes include frequent urination, excessive thirst, unexplained weight loss, extreme hunger, sudden vision changes, tingling or numbness in hands or feet, frequent tiredness, very dry skin, slow healing of sores and a proclivity toward more infections than usual. Nausea, vomiting, or stomach pains may accompany some of these symptoms.

There are three types of diabetes. Type 1 diabetes, Type 2 diabetes, and Gestational diabetes. Each one is briefly described.

(1) Type 1 diabetes

It was previously called insulin-dependent diabetes mellitus (IDDM). Type 1 diabetes may account for $5 \%$ to $10 \%$ of all diagnosed cases of diabetes. Risk factors are less well defined for type 1 diabetes than for Type 2 diabetes. Genetic and environmental factors are involved in the development of this type of diabetes.

Proceedings of the 2001 American Society for Engineering Education Annual Conference \& Exposition Copyright @ 20001, American Society for Engineering Education 
(2) Type 2 diabetes

It was previously called non-insulin-dependent diabetes mellitus (NIDDM) or adult-onset diabetes. Type 2 diabetes may account for about $90 \%$ to $95 \%$ of all diagnosed cases of diabetes. Risk factors for Type 2 diabetes includes older age, obesity, family history of diabetes, prior history of gestational diabetes, impaired glucose tolerance, physical inactivity, and race/ethnicity.

(3) Gestational diabetes:

It develops in $2 \%$ to $5 \%$ of all pregnancies but usually disappears when a pregnancy is over. Pregnant women have enough insulin, but the effect of insulin is partially blocked by variety of other hormones produced in the placenta. This condition is called insulin resistance.

\subsection{Treatment for Diabetes:}

Treatment of diabetes is aimed at keeping blood glucose near normal levels at all times. Training in self-management is integral to the treatment of diabetes. Treatment must be individualized and must address medical, psychosocial, and lifestyle issues.

Lack of insulin production by the pancreas makes Type 1 diabetes particularly difficult to control. Treatment requires a strict regimen that typically includes a carefully calculated diet, planned physical activity, home blood glucose testing several times a day, and multiple daily insulin injections.

Treatment of Type 2 diabetes typically includes diet control, exercise, home blood glucose testing, and in some cases, oral medication and/or insulin. Approximately $40 \%$ of people with Type 2 diabetes require insulin injections.

The causes of Type 1 diabetes appear to be much different than those for Type 2 diabetes, though the exact mechanisms or development of both diseases are unknown. The appearance of type 1 diabetes is suspected to follow exposure to an environmental trigger, such as an unidentified virus, stimulating an immune attack against the beta cells of the pancreas (that produce insulin) in some genetically predisposed people.

A number of studies have shown that regular physical activity can significantly reduce the risk of developing Type 2 diabetes. It also appears to be associated with obesity. Researchers are making progress in identifying the exact genetics and triggers that predispose some individuals to develop Type 1 diabetes, but prevention, as well as a cure, remains elusive. Three approaches could be examined to take care for patients with diabetes. These are: prevent diabetes, cure diabetes, and take better care of people with diabetes to prevent devastating complication.

Several approaches to cure diabetes are being pursued like Pancreas transplantation, Islet cell transplantation (islet cells produce insulin), artificial pancreas development, genetic manipulation by inserting insulin gene for people with Type 1 diabetes. All the above stated approaches have a long way to go, as they need thorough research and testing. Early diagnosis and prevention seems to be the best approach. This is where computer science and medicine fields can come together to help patients.

Proceedings of the 2001 American Society for Engineering Education Annual Conference \& Exposition Copyright@20001, American Society for Engineering Education 


\section{Computer-Aided Diagnosis}

Due to rising cost of health care, medical software has proved useful for assisting medical practitioners and helping patients to self manage diabetes. Several software tools are available that mostly help in managing stable levels of glucose throughout the day. A few of these are mentioned below:

AIDA ${ }^{2}$ contains a simple model of glucose-insulin interaction in the human body. It is intended for simulating the effects on the blood glucose profile of changes in insulin and diet for a typical insulin-dependent diabetic patient (IDDM).

BG Tracker ${ }^{3}$ is an application designed to help patients manage diabetes. This program accepts point data from a blood glucose curve and outputs a graph of the data to the screen.

D-Net Blood Glucose Monitoring ${ }^{4}$ allows patients to create personal blood glucose monitoring database on-line. Patients can enter daily blood glucose values, get graphic feedback of daily blood glucose values over a number of days, and check the values they have entered in database.

The Diabetes Research online glucometer project ${ }^{5}$ is a free resource designed to assist in the self-management of diabetes.

The MyDiabetes Web site ${ }^{6}$ has a personal "Daily Diary" for tracking blood glucose levels, medication usage, diet, exercise and care schedule.

The Diabetic Daily Log (TDDL) ${ }^{7}$ tracks daily activities of a diabetic. It records glucose test results, insulin medication, weight, blood pressure, exercise, meals, snacks, and other general comments.

DIABASS (Diabetes Assistant) ${ }^{8}$ It produces graphs and statistical analysis of insulin dosages and blood sugars.

Diabetes Mentor ${ }^{9}$ It computerizes and graphs diabetes-related measurements, helping patient adjust regime to achieve targeted blood glucose.

Diabetes Tracker ${ }^{10}$ It is designed specifically for reporting on blood glucose levels.

DiaTrends ${ }^{11}$ is designed for health care professionals to help track and improve diabetes management outcomes.

HealthDesk OnLine for Diabetes ${ }^{12}$ records and analyzes glucose readings. It can be used to plan and track nutrition, exercise and health history. 
HealthView for Diabetes ${ }^{13}$ provides information on how people with diabetes can use the software to improve their diabetes therapy.

Mellitus Manager ${ }^{14}$ The software provides analysis tools to help patients get a handle on blood sugar readings.

\section{Artificial Intelligence and Diabetes}

The medical field was one of the first testing grounds for Artificial Intelligence (Expert Systems) technology. MYCIN ${ }^{15}$, NURSExpert ${ }^{1}$, CENTAUR, DIAGNOSER, MEDI and GUIDON ${ }^{16}$, MEDICS ${ }^{17}$, and DiagFH ${ }^{18}$ are few of the first and successful medical Expert System. According to Jackson ${ }^{19}$ an Expert System is a computer program that represents and reasons with knowledge of some subject specialist with a view to solving problems or giving advise.

Diabetes is a disease that affects many people in the world ${ }^{1}$. Diabetes cost in the U.S.A. is estimated to represent $5.8 \%$ of total personal health-care expenditures. In Europe diabetes is estimated to consume about $10 \%$ of the total health care budget. It is evident according to these statistics that something has to be done to help people with diabetes. As was discussed in the previous section, there are several software tools that can be used by people with diabetes. Most of these tools are used to keep track of the sugar level in the blood. No tool was found that could help people in the decision making process according to the blood sugar level. An Intelligent Diabetes Management System was developed by computer science students at Texas A\&M University Corpus Christi to help diabetics people to monitor and to control the blood glucose level.

\section{Implementation}

The procedure used for the implementation of an expert system is composed of the following phases: 1.Knowledge acquisition. 2. Knowledge representation. 3. Coding, and 4. Testing. Each phase is described below.

\subsection{Knowledge Acquisition (KA)}

Knowledge Acquisition (KA) is the transfer of problem solving expertise from some knowledge source to computer code. The KA for this project consisted of several interviews with diabetes experts Dr. Steve Ponder and Dr. Hilda Ramirez from Driscoll's children Hospital. Different diabetes type 2 problems and solutions were explained to the students. After each interview the students analyzed the information and wrote questions to ask the diabetes experts during the next interview. The knowledge acquisition phase lasted for three months.

\subsection{Knowledge Representation}

Once the knowledge acquisition phase was over, the knowledge was represented in a form that could be read by a computer. Rules were used to represent knowledge. A rule has two components: IF < situation> THEN < suggestion>. The IF-part (antecedent/left hand side) 
describes a situation under which the rule is applicable. The THEN part (consequent/right hand side) suggests an action to be performed under the situation (for action rules) or a plausible inference to make under the situation (for inference rules).

\subsection{Coding}

The Expert System was implemented using WxClips ${ }^{20,21,22}$ as the development tool. WxCLIPS(Windows C Language Integrated Production System) was developed at NASA, Johnson Space Center and is public domain software. The basic components of WxCLIPS are shown in Figure 1. Each one is described briefly.

- User Interface: It is the mechanism by which the user and expert system communicate.

- Fact-List: It is a global memory of data stored in WxCLIPS syntax as shown in Figure 2.

- Knowledge-base: It contains all rules used by the expert system. The Rule(s) syntax is presented in Figure 3 and an example rule is shown in Figure 4.

- Inference engine: It makes inferences by deciding which rules are satisfied by facts, prioritizes the satisfied rules, and executes the rule with highest priority.

- Agenda: A prioritized list created by the inference engine of instances of rules whose patterns are satisfied by facts in the fact list. An example is shown in Figure 5.

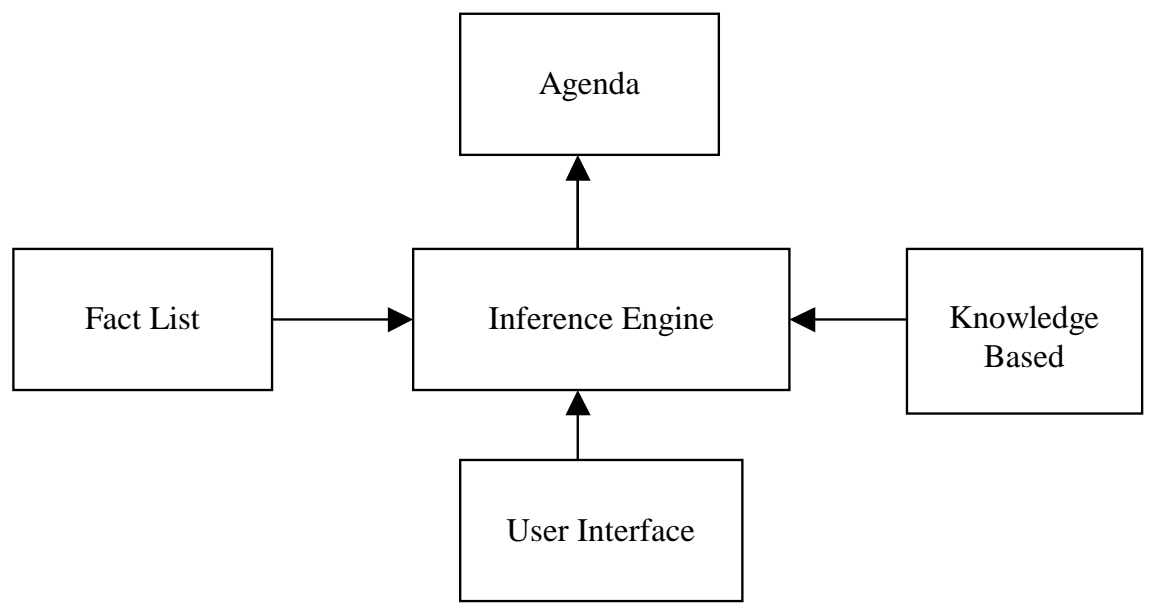

Figure 1. WxCLIPS Architecture

English:

The symptom of the patient pertaining Diabetes.

CLIPS:

(deffacts patient "Patient's Symptoms"

( report Blood Pressure above 130)

( report Blood Glucose between 130-140 mg/dl )

( report Frequent Tiredness ) )

Figure 2. Fact List

Proceedings of the 2001 American Society for Engineering Education Annual Conference \& Exposition

Copyright@ 20001, American Society for Engineering Education 


\section{Testing}

For a real world project, the system should be tested to determine whether it provides good quality decisions. The reasoning technique should be tested for correctness; it should be checked for attaining the desired accuracy rate. Testing helps check omitted rules, incorrect rules, overlooked special cases. It also allows checking the correctness of association of rules and missing useful relationships. Exhaustive testing will verify that all paths in your knowledge base are covered. The Expert System was tested by Dr. Ponder and Dr. Ramirez. The Prototyping approach ${ }^{19}$ was used to implement the Intelligent Diabetes Management System.

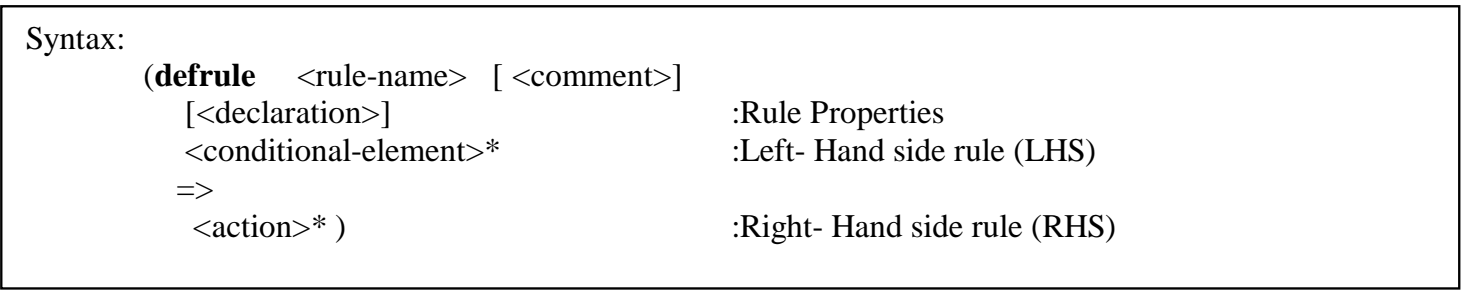

Figure 3. Rule Syntax

A small domain of the knowledge acquired was implemented in WxCLIPS and presented to the diabetes experts. They tested the prototype using different scenarios. They recommended additions, changes or deletions from the conclusions given by the Intelligent Diabetes Management System. Once the diabetes experts agreed with the recommendations given by the expert system, a new prototype with more knowledge was developed and presented again to the experts for testing. The procedure continued until all the acquired knowledge was included in the Intelligent Diabetes Management System knowledge base.

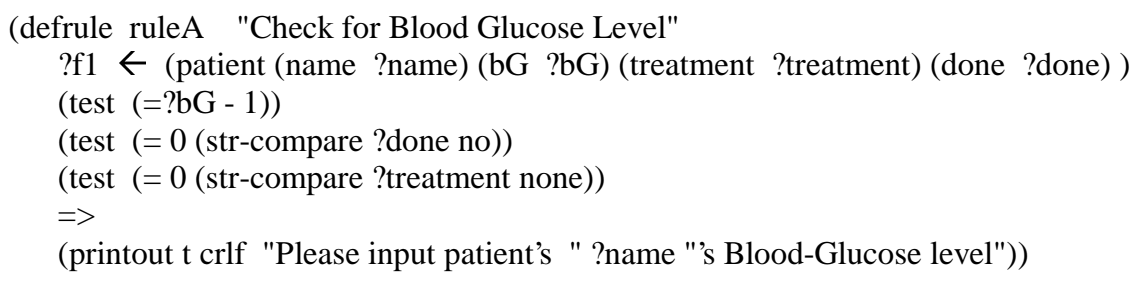

Figure 4. Example of a rule

\section{Expert System Recommendations}

There is an interaction between the Intelligent Diabetes Management System and the diabetic person (expert system user). The Intelligent Diabetes Management System will ask a question and the user will answer. This procedure will continue until the Intelligent Diabetes Management System has enough information to give a recommendation. There are several categories of questions. Some of these are: 1 . Personal information questions such as age, gender, and weight. 2. Questions to detect diabetes such as; "Is the person hungry?"; "Is the 
person thirsty?"; "Is the person always tired?"; "Has the person blurry vision?"; "Has the person dry skin?" etc. 3. Questions about what the person eats such as bread (how many slices?); milk (how many cups?), vegetables (how many cups?); fruits (how many cups?), etc. 4. Questions about the blood sugar level $(<60,60-100,101-125,126-200,201-240,>240) 5$. General diabetes related questions such as where the person stores the insulin bottles, who gives the insulin shots (if person is < than 18 years old), etc.

Once the Expert System has enough information it will display its recommendations to maintain the glucose level in the blood within acceptable values. The following is an example: According to the glucose level in your blood and your diet today, you need to take 5 units of insulin.

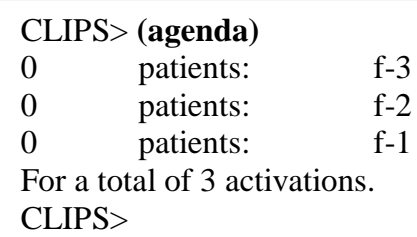

Figure 5. Agenda

\section{Conclusions}

The Intelligent Diabetes Management System is the result of two research projects: The first phase was implemented by students taken Artificial Intelligence during the fall 1999. In this phase the students did a literature review, including software tools applied to diabetes. They presented a small prototype in CLIPS 6.0 as a final project. The second phase was implemented for students of an Expert System course in Spring 2000. The students worked for more than three months learning about diabetes and doing the knowledge acquisition interviewing Dr. Ponder and Dr. Ramirez. The system was implemented in WxCLIPS because it has a more friendly user interface than CLIPS 6.0.

By using the Intelligent Diabetes Management System, patients can enter their symptoms any time and get diagnosis about their blood sugar levels. Medical trainees can use this software to study about diabetes. Thus, this tool proves advantageous for both testing and training purposes.

\section{1 Future Work}

The Intelligent Diabetes Management System is still a small prototype that can be extended by other students. Knowledge acquisition is needed to help people with diabetes type 1, and Gestational diabetes. This knowledge can be easily incorporated in the expert system knowledge base. One of the priorities that we have is the translation of the system to Spanish because more than $50 \%$ of our community is of hispanic origin. 
Once the Intelligent Diabetes Management System has enough knowledge about the three types of diabetes, Dr Ponder wishes to install the software in computers that will be outside the waiting room at Driscoll Children's Hospital. He believes that patients can learn more about diabetes while waiting for the doctor. Also in the future the Intelligent Diabetes Management System will be available on the WEB so that people everywhere can check their blood sugar level and learn more about diabetes using a PC. The system is portable since WxClips can run on most popular platforms like Windows, Sun, Linux etc.

Bibliography

[1] O. HeJlesen, S. Plogmann, and D. Cavan. DiasNet - An Internet Tool for Communication and Education in Diabetes. International Symposium on Computer and Diabetes Care. Rochester MN, September 8-10, 2000

[2] AIDA educational version for Diabetes. http://www.diabetic.org.uk/

[3] BG Tracker educational version - http://www.bgtracker.com

[4] D-Net Blood Glucose Monitoring version - http://dnet.ori.org/newuser

[5] Diabetes Research online project version - http://www.diabetesresearch.com/

[6] MyDiabetes healthcare version - http://www.mydiabetes.com/

[7] The Diabetic Daily Log reference - http://members.aol.com/kennzo

[8] FIT foundation Diabetes - http://diabtrends.com/fitenone.htm

[9] Vigora Diabetes healthsite - http://www.vigora.com/

[10] Diabetes Tracker reference http://www.prihar.com/

[11] DiaTrends reference - http://overlooksoftware.com/

[12] HealthDesk OnLine reference - http://www.healthdesk.com/

[13] HealthView for Diabetes reference - http://www.healthviewdiabetes.com/

[14] Mellitus Manager reference http://www.metamedix.com/

[15] Buchanan B, Shortliff, E. Rule Based Expert Systems. The Mycin Experiments of the Stanford Heuristic Programming Project. Addison-Wesley. 1984

[16] MMWR Weekly publications, Diabetes Preventive-

Care Practices in Managed-Care Organizations, October 29,1999.

[17] Fieschi, M., Artificial Intelligence in Medicine, Chapman and Hall, London, 1990.

[18] Joslin Diabetes Center publications, Goals for Blood Sugar Control http://www.joslin.harvard.edu/eduaction/

[19] Jackson, P. Introduction to Expert Systems. Third Edition. Addison-Wesley, 1998

[20] NASA, Lyndon B. Johnson Space Center, CLIPS Basic Programming Guide, 1991.,Houston, TX.

[21] Martin, Linda and Taylor, Wendy. A booklet of CLIPS Applications, NASA, Johnson Space Center, Houston, TX, 1992.

[22] CLIPS, A Tool for Building Expert Systems, http://www.ghg.net/clips/.

\section{MARIO A GARCIA}

Mario A Garcia is an assistant professor at Texas A\&M University Corpus Christi. Dr. Garcia received a B.S. degree in Electrical Engineering from Tecnologico de Saltillo, Mexico, He received a M.S. in Electrical Engineering from Tecnologico de la Laguna, Mexico, He recived a M.S. in Artificial Intelligence from ITESM, Mexico, and his a Ph.D. in Computer Science from Texas A\&M.

\section{CARL STEIDLEY}

Carl Steidley is Professor of Computer Science and Chair of Computing and Mathematical Sciences. His interests are in the applications of artificial intelligence, real-time computing, and robotics. He taught computer science at Southeastern La. Univ., Central Washington Univ., Austin Peay State Univ., and Oregon Institute of Technology. He has research at NASA Ames Research Center, Oak Ridge Natl. Labs, and Electro Scientific Industries in Portland 